\title{
SUB-MICROSCOPIC LOCALIZATION OF MINERALS IN SKELETAL MUSCLE BY INTERNAL 'MICRO-INCINERATION' WITHIN THE ELECTRON MICROSCOPE
}

\author{
By Dr. M. H. DRAPER* \\ Physiology Department, University of Adelaide \\ AND
}

A. J. HODGE

Division of Industrial Chemistry, Council for Scientific and Industrial Research, Malbourne

$\mathrm{R}$ ECENT work on contractile phenomena in muscle 1 indicates that a complete understanding of these processes demands a fuller knowledge of the sub-mieroscopic morphology and distribution of the mineral content. An electron microscopic study of pruscle has therefore been undertaken as part of a general investigation of fibrous proteins ${ }^{2}$ in progress in this laboratory. The present communication represents a part of the investigation which should be of immediate interest to workers in this field.

The morphology of skeletal muscle has been described within the limits of optical resolution by numerous workers ${ }^{3}$. The main structural features and the 'reversal of striation' phenomenon, which gives rise to contraction bands, have been confirmed by recent electron microscopical studies ${ }^{4}$. Knowledge of the distribution of mineral content has been derived from staining and micro-incineration techniques $^{3}$, and more recently by the application of the emission electron microscope ${ }^{5}$, whereby it is possible to differentiate potassium and sodium from calcium and magnesium. However, the limited resolving power available from these methods did not allow precise location of the mineral content on a submicroscopic scale. The method described here has enabled us to locate inorganic matter with a precision of approximately $100 \mathrm{~A}$.

In applying the new technique, we have employed suspensions of myofibrils obtained from formalinfixed toad and rabbit leg muscles, which are structurally very similar. The term 'myofibril' as used here refers to fragments of tissue rather than the intact myofibril of the classical morphologist. Fig. 1 is an electron micrograph of a myofibril stained with phosphomolybdic acid, which effectively increases the over-all contrast and enhances certain features of the myofibrils. In the examination of muscle we have found this reagent more suitable then phosphotungstic acid, which tends to 'stain' the myofibrils too deeply owing to the higher electron-scattering power of tungsten atoms. The $Z, M, Q$ (or $A$ ), $H, J$ (or $I$ ) and $N$ bands are clearly visible, as are the four fine bands lying, two on either side of and adjacent to the $M$ band, within the $H$ band. The latter fine bands have not previously been described and their significance is unknown. We have found, in agreement with the work of Hall, Jakus and Schmitt4, that the myofibrils contain longitudinal filaments, presumably of actomyosin, which are aligned parallel and extend continuously through all bands in both the extended and contracted states. There is some evidence to suggest that the myofibrils are thin sheets of this fibrous material, which in the intact muscle are folded about the long axis and embedded in bundles of collagen protofibrils. This thesis affords an explanation of the irregular shapes and variable

* Junior Research Fellow, National Health and Medical Research Council.

sizes of myofibrils as seen in transverse section. Another morphological feature visible in Fig. 1 is the periodic darkly staining transverse striation which appears throughout the length of the sarcomere. The spacing is about $400 \mathrm{~A}$. and varies inversely with the degree of contraction within the limits of error arising from fortuitous stretching of contracted myofibrils during preparation of the suspensions. Fig. 2 shows unstained myofibrils which have been shadow-cast with platinum. The fine periodic striation is par. ticularly evident, and it is to be noted that it is a feature of the individual actomyosin filaments. The thinness of the material is evident from the small shadow-length.

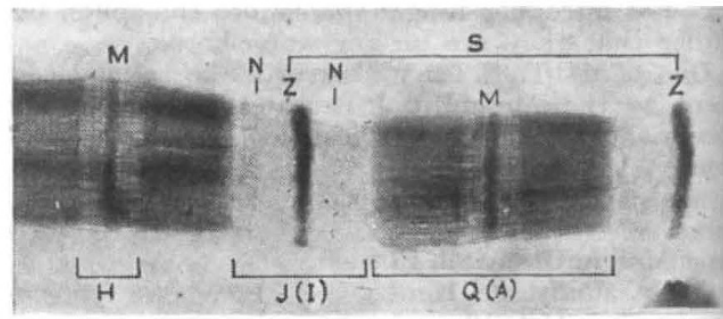

Fig. 1. Myoflbril from formalin-fixed toad skeletal muscle, stained with phosphomolybdic acid. $20,000 \times$ Positive print (that is, enlarged from original plate as negativè)

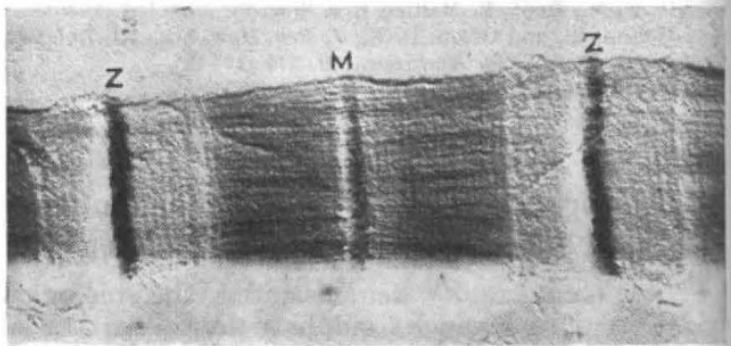

Fig. 2. Myoflbril from formalin-flxed toad skeletal muscle, shadow-cast with $10 \mathrm{~A}$. thickness of platinum; shadow ratio approximately $4: 1$; printed with shadows white. 20,000 Positive print

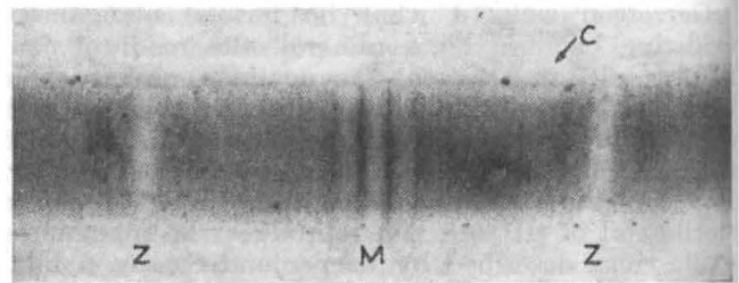

Fig. 3. Myofibril from formalin-fixed rabbit skeletal muscle after exposure to high-intensity electron beam in the electron micro. scope. 20,000 $\times$ Positive print

$S$, sarcomere

$Q(A), Q$ band or anisotropic band

$J(I), J$ band or isotropic band

$Z, Z$ band or telophragma
$M, M$ band or mesophragma $\ddot{H}, \boldsymbol{H}$ band $N, N$ band $C$, collagen protoflbril 
The success of the 'micro-incineration' technique depends on the ability to attain high current densities through the specimen, a condition which was easily satisfied by the use of a cathode-biased electron gun on our R.C.A. Model $E M U$ electron microscope. It was observed that unstained myofibrils, when suddenly subjected to a high-intensity beam in the electron microscope, suffered a serious decrease in contrast, presumably resulting from the loss of bound water, organic matter and salts volatile under electron bombardment. The recent observation ${ }^{6}$ that the evaporation of potassium salts under high. intensity beams in the electron microscope may not be an entirely thermal phenomenon has led us to believe that our observation was of an essentially similar nature. However, in a few fields containing myofibrils of favourable dimensions, the structure of the residue was particularly clear. Fig. 3 is an electron micrograph of such a field. A striking feature of the phenomenon is the complete disappearance of $Z$ and $M$ bands. This has been observed to occur in a large number of myofibrils. However, the fine transverse striation was only obtained when the optimum conditions of myofibril dimension and rate of increase of beam intensity were satisfied. The periodicity of these bands of residual crystalline material was again about $400 \mathrm{~A}$., leaving little doubt that they correspond with the striations present in both stained and unstained myofibrils viewed with low beam-intensity (Figs. 1 and 2). The relatively refractory nature of the residual crystalline material suggests that it consists almost entirely of salts of magnesium and calcium, a finding which is supported by the thermal stability and relative resistance to electron bombard. ment exhibited by crystals of these salts. The ease with which potassium salts may be evaporated under similar conditions justifies the conclusion that all the potassium content of the myofibrils was evaporated under the influence of the beam.

Since we have invariably used suspensions of the fixed material obtained by mechanical agitation and afterwards centrifuged and resuspended several times in distilled water, it must be concluded that the residual mineral content of the myofibrils is either bound by the protein or present in the form of insoluble salts. The disappearance of the $Z$ and $M$ bands and of the $Q$ substance during 'micro-incineration' (Fig. 3) indicates that magnesium and calcium are either absent, or present only in very small amount in these components. Furthermore, their content is distributed periodically throughout the sarcomere.

The distribution of potassium is less definite than that of magnesium and calcium. Macallum ${ }^{7}$ obtained a distribution of potassium resembling the distribution of density in Fig. 1. The distribution of mineral ash as demonstrated by Scott $^{8}$ was similar, the $J$ and $H$ bends being almost ash-free, while the $Q$ and $Z$ bands were represented by deposits of ash. It is to be noted that the thickness variation of the dehydrated myofibrillar fragments, as evident from the shadow in Fig. 2, corresponds exactly with this ash distribution. Preliminary experiments have shown that the myofibrils in our suspensions contain some potassium, amounting to about 0.05 per cent of the dry weight, and apparently firmly bound by the protein. The disappearance of the $Z$ and $M$ bands and of the $Q$ substance during high-intensity electron bombardment then suggests that the mineral content of these components consists largely of potassium and perhaps sodium.
This conclusion is interesting when compared with the affinities for phosphotungstic acid exhibited by the various bands. Although this reagent is used in the analytical determination of potassium ${ }^{9}$, it is known to react with proteins and protamines, and cannot be regarded as specific for the localization of potassium. However, the low affinity of the $J$ and $H$ bands and the high affinity of the $Z, M$ and $Q$ bands for this reagent indicates that at least a part of its 'staining' action on muscle is attributable to combination with potassium held by the protein.

With these observations in mind, the ultrastructure of skeletal muscle begins to assume a concrete form. The myofibril consists of long filaments of actomyosin, aligned parallel in thin sheets, the filaments being held together by the transverse $Z$, $M$ and lesser bands and perhaps also by an interfilamentous matrix substance. A regular fine transverse striation is present arising from a periodic concentration of magnesium and calcium bound to the actomyosin framework, the periodicity being about $400 \mathrm{~A}$. Superimposed on this skeletal framework is the $Q$-substance, which presumably contains protein in globular form, potassium, chloride, phosphate, adenosine triphosphate and other constituents, and which moves in characteristic fashion within the sarcomere during contraction. While it is clear that $Z$ and $M$ bands exercise a structural function in binding the filaments together, it seems likely that they perform other functions also, possibly connected with innervation of the myofibrils.

The new technique has also been successfully applied in the determination of mineral distribution in collagen protofibrils, one of which may be discerned in Fig. 3. The minute residue is concentrated in the narrow portions of the collagen repeating-units.

Further work is proceeding on the localization of mineral content of muscle, collagen and other fibrous protein tissues. A fuller account will be published elsewhere.

1 Szent-Györgyi, A., "Chemistry of Muscular Contraction" (New York, Academic Press, 1947).

Farrant, J. L., Mercer, E. H., and Rees, A. L. G., Nature, 159, 535 (1947).

8 Jordan, H. E., Physiol. Rev., 13, 301 (1933).

' Hall, C. E., Jakus, M. A., and Schmitt, F. O., Biol. Bull., 90, 32 (1946). Scott, G. H., and Packer, D. M., Anat. Rec., 74, 31 (1939).

- Burton, E. F., Sennett, R. S., and Ellis, S. G., Nature, 160, 565 (1947).

' Macallum, A. B., J. Physiol., 32, 98 (1905).

S Scott, G. H., Proc. Soc. Exp. Biol. Med., 29, 349 (1932).

- Rieben, W. K., and van Slyke, D. D., J. Biol. Chem., 156, 765 (1944).

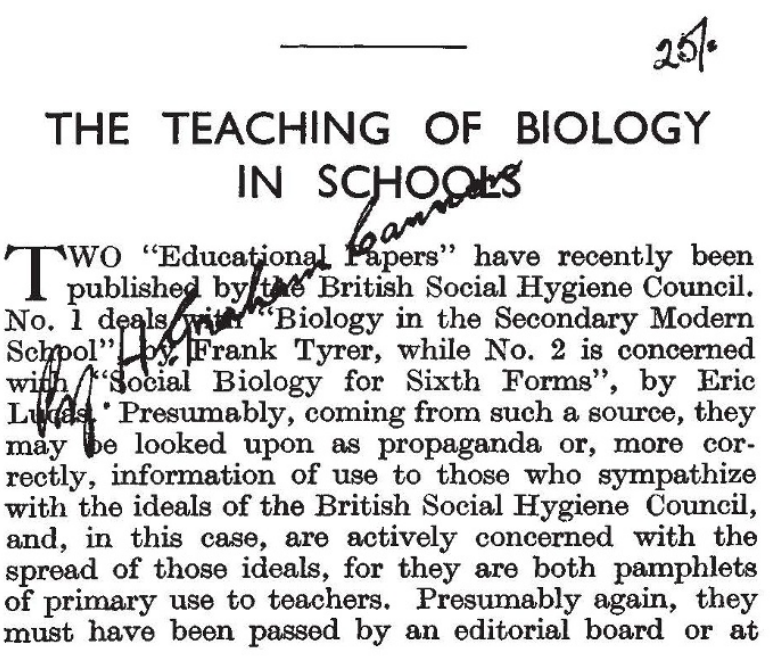

